\title{
Sु \\ Symmetry-breaking Fermi surface deformations from central interactions in two dimensions
}

\author{
Jorge Quintanilla, ${ }^{1, *}$ Masudul Haque, ${ }^{2}$ and A. J. Schofield ${ }^{3}$ \\ ${ }^{1}$ ISIS Spallation Facility, STFC Rutherford Appleton Laboratory, Harwell Science and Innovation Campus, Didcot OX11 OQX, \\ United Kingdom \\ ${ }^{2}$ Max Planck Institute for Physics of Complex Systems, Nöthnitzer Strasse 38, Dresden, Germany \\ ${ }^{3}$ School of Physics and Astronomy, University of Birmingham, Birmingham B15 2TT, United Kingdom
}

(Received 10 June 2008; revised manuscript received 25 June 2008; published 29 July 2008)

\begin{abstract}
We present a mean-field theory of the Pomeranchuk instability in two dimensions, starting from a generic central interaction potential described in terms of a few microscopic parameters. For a significant range of parameters, the instability is found to be pre-empted by a first-order quantum phase transition. We provide the ground-state phase diagram in terms of our generic parameters.
\end{abstract}

DOI: 10.1103/PhysRevB.78.035131

PACS number(s): 71.10.Hf, 71.10.Ay, 71.30.+h

\section{INTRODUCTION}

A central theme in the study of strongly correlated electron systems is the appearance of novel types of ordering and phase transitions leading to such unconventionally ordered states. Phases with liquid crystalline symmetry have emerged as an intriguing theme. They have been studied and proposed, for example, in the context of quantum Hall systems, ${ }^{1-8} s$-wave pairing of polarized fermions, ${ }^{9}$ and in Hubbard-like models. ${ }^{10-15}$ One prominent mechanism for such phases is via Pomeranchuk instabilities, which are distortion instabilities of the Fermi surface. ${ }^{16}$ A Pomeranchuk instability occurs in the angular-momentum channel $l$ when the corresponding Fermi liquid parameter $F_{l}$ is sufficiently negative. ${ }^{16}$ The phase resulting from an $l=2$ type instability is a nematic, because the orientation symmetry of the continuum or the orientation symmetry of the lattice point group is broken, modulo an inversion symmetry, while translational symmetry remains unbroken. Pomeranchuk instabilities have received significant attention recently, both in the continuum ${ }^{17-23}$ and lattice ${ }^{15,24-27}$ contexts.

In this paper we focus on continuum systems, where the Pomeranchuk instability breaks a continuous symmetry. This is particularly relevant to ultracold fermionic gases and lowdensity two-dimensional (2D) electron systems. The Hamiltonian most prominently studied for this case has been of the quadrupole-quadrupole type explicitly designed in Ref. 17 to produce an $l=2$ instability. ${ }^{21-23}$ It is therefore important to study Pomeranchuk instabilities arising from more generic Hamiltonians. In a previous paper, two of the present authors studied shape deformations of the Fermi surface of a threedimensional system, arising from central interactions. ${ }^{18}$ In particular, Ref. 18 finds that a central interaction, if it has a sharp feature at a finite length scale $r_{0} \gtrless k_{F}^{-1}$, can cause deformations of the Fermi surface. Nonmonotonic "deltashell," and monotonic "hard-core" repulsive potentials were analyzed, and they were all found to lead to the effect. A screened Coulomb interaction, on the other hand, was found not to lead to a Pomeranchuk instability.

In this paper we provide a mean-field treatment of two dimensions, where much of the current interest lies. In the interest of providing generic results, we will parametrize our central interaction $V(r)$ by a small number of parameters, namely the values of angular-momentum components of the potential and its momentum-space derivatives at the Fermi momentum. These turn out to be the essential parameters for the description of Fermi surface shape distortion transitions. A phase diagram in terms of these parameters provides much more general information than the consideration of particular forms of $V(r) .{ }^{18}$ Our framework has the added advantage that a single description treats not only the transition in the $l=2$ channel, but in every angular momentum channel $l>1$. In other words, we in fact present a mean-field theory not only for distortions leading to nematic symmetry, but also to the others shown in Fig. 1.

One important result of our analysis is that we find the shape-deformation transitions to be of first order for significant regions of parameter space. This implies that in many realistic cases, where one might get shape deformation instabilities of the Fermi surface, the transition is discontinuous and does not involve quantum critical behavior.

We introduce the model in Sec. II and the mean-field treatment in Sec. III. The details of the theory are worked out in the next three sections. Sec. VII describes the parameter regimes where we have first-order transitions. In Sec. IX we provide a comparison with the Hamiltonian of Ref. 17, which is the dominant Hamiltonian used in the recent literature for the continuum $l=2$ Pomeranchuk transition. Finally, in Sec. VIII we use our mean-field theory to compute the Landau Fermi liquid parameters in terms of the microscopic interaction potential. In the final section we lay out our conclusions.

\section{MODEL}

We consider the following continuum Hamiltonian, describing interacting fermions in two dimensions:

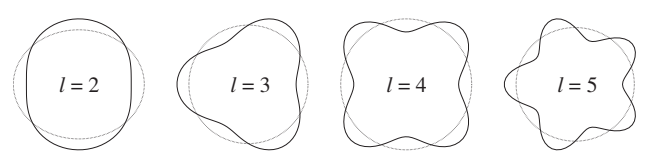

FIG. 1. Shape of Fermi surface of 2D continuum system, before (thinner line) and after (thicker line) a Pomeranchuk instability. Several possible symmetries $(l=2,3,4,5)$ are shown for the Pomeranchuk order. 


$$
\begin{aligned}
H= & \int d \mathbf{r} \psi_{\mathbf{r}}^{\dagger}\left[\epsilon_{\nabla_{\mathbf{r}^{i}} i}-\mu\right] \psi_{\mathbf{r}}+\frac{1}{2} \int d \mathbf{r} \int d \mathbf{r}^{\prime} \\
& \times V\left(\left|\mathbf{r}-\mathbf{r}^{\prime}\right|\right) \psi_{\mathbf{r}}^{\dagger} \psi_{\mathbf{r}^{\prime}}^{\dagger} \psi_{\mathbf{r}^{\prime}} \psi_{\mathbf{r}} .
\end{aligned}
$$

Here $\epsilon_{\hat{\mathbf{p}} / \hbar}=\hat{\mathbf{p}}^{2} / 2 m$ gives the "bare" dispersion relation in terms of the momentum operator $\hat{\mathbf{p}} \equiv(\hbar / i) \nabla_{\mathbf{r}}$ (free fermions of mass $\mathrm{m}) . V(r)$ is a central interaction potential. For simplicity we consider spinless fermions. (In any case, when the interaction is spin independent, the Pomeranchuk instability will be degenerate in the spin channels. ${ }^{18}$ On the other hand for spin-dependent interactions there can be emergent spinorbit coupling ${ }^{28}$ and nontrivial spin configurations in momentum space. ${ }^{29}$ ) In reciprocal space, the Hamiltonian [Eq. (1)] takes the form

$$
\begin{aligned}
H= & \sum_{\mathbf{k}}\left(\epsilon_{\mathbf{k}}-\mu\right) \psi_{\mathbf{k}}^{\dagger} \psi_{\mathbf{k}}+\frac{1}{2 \Omega} \sum_{\mathbf{k}, \mathbf{k}^{\prime}, \mathbf{q}} V(\mathbf{k} \\
& \left.-\mathbf{k}^{\prime}\right) \psi_{\mathbf{q} / 2-\mathbf{k}^{\prime}}^{\dagger} \psi_{\mathbf{q} / 2+\mathbf{k}^{\prime}}^{\dagger} \psi_{\mathbf{q} / 2+\mathbf{k}} \psi_{\mathbf{q} / 2-\mathbf{k}},
\end{aligned}
$$

where $\psi_{\mathbf{k}}^{\dagger}=\int d^{2} \mathbf{r} \Omega^{-1 / 2} e^{i \mathbf{k} . \mathbf{r}} \psi_{\mathbf{r}}^{\dagger}$ and $V(\mathbf{k}) \equiv \int d^{2} \mathbf{r} e^{-i \mathbf{k} \cdot \mathbf{r}} V(r)$. The area of the sample is $\Omega$.

There is a large body of literature on behaviors that can emerge from Hamiltonians of the type given by Eq. (2). For the attractive case see, for example, the discussions of unconventional superconductivity, Bose-Einstein condensation of "preformed pairs" and "fermion condensation" in Refs. 30-33. Here we are concerned with the emergence of anisotropy of the Fermi surface due to repulsive interactions. As in the case of anisotropic pairing in superconductors, the key is the dependence of the interaction strength $V(\mathbf{q})$ on momentum transfer, $\hbar \mathbf{q}$. In the case of a contact potential, $V(\mathbf{q})$ $=$ constant, the only possibilities are isotropic: a conventional Bardeen-Cooper-Schrieffer (BCS) instability for attractive interactions or a quantum gas-liquid transition for repulsion.

With more general momentum dependence, the Hamiltonian [Eq. (2)] can lead to an anisotropic state through a Pomeranchuk instability. The relevant order parameter is

$$
\left\langle\hat{b}^{\dagger}(\phi)\right\rangle \equiv \sum_{k} \cos \left(l \theta_{k}\right)\left\langle\psi_{k}^{\dagger} \psi_{k}\right\rangle,
$$

where $l=2,3,4, \ldots$, leading to the Fermi surface shape deformations represented in Fig. 1 . The $l=1$ instability is forbidden in a Galilean-invariant system. ${ }^{18,34}$

Pomeranchuk order can be described as particle-hole pairing with center-of-mass momentum $\mathbf{q}=0$ but finite internal angular momentum quantum number, $l>0$. In this sense, it is the analog of "exotic" superconductivity, where $l>0$ pairing occurs in the particle-particle channel. Indeed that analog has been shown recently by one of us to extend quantitatively to the disorder dependence of a Pomeranchuk transition temperature. ${ }^{35}$

It is useful to introduce the partial wave decomposition of the interaction potential:

$$
V\left(\mathbf{k}-\mathbf{k}^{\prime}\right)=\sum_{l=0}^{\infty} V_{l}\left(k, k^{\prime}\right) \cos \left[l\left(\theta_{\mathbf{k}}-\theta_{\mathbf{k}^{\prime}}\right)\right],
$$

where $k=|\mathbf{k}|$, etc. The amplitudes $V_{l}$ are

$$
V_{l}\left(k, k^{\prime}\right)=\left(1+\delta_{l, 0}\right) 4 \pi \int_{0}^{\infty} d r r J_{l}(k r) J_{l}\left(k^{\prime} r\right) V(r) .
$$

[Note that $V(k, q)=V(q, k)]$. We provide below a mean-field theory that is applicable to any Hamiltonian of the form of Eq. (2). The theory is quite independent of the details of the interaction potential $V\left(\left|\mathbf{r}-\mathbf{r}^{\prime}\right|\right)$, but depends only on a few parameters derived from it. In particular, we will use: (i) the values of the amplitudes $V_{l}$ on the Fermi surface and (ii) their derivatives on the Fermi surface. These are the parameters $V^{(n)}$ introduced in Eq. (21).

\section{MEAN-FIELD THEORY}

Our mean-field theory is based on the following ansatz for the ground state:

$$
\left|\Lambda_{\mathbf{k}}\right\rangle=\prod_{\mathbf{k}}\left[\Theta\left(\varepsilon_{\mathbf{k}}\right)+\Theta\left(-\varepsilon_{\mathbf{k}}\right) \psi^{\dagger}(\mathbf{k})\right]|0\rangle .
$$

Here $|0\rangle$ is the vacuum and $\psi^{\dagger}(\mathbf{k})$ creates an electron in a plane-wave state. The ansatz wave function is a Slater determinant of plane waves. $\varepsilon_{\mathbf{k}}$ is an arbitrary dispersion relation. Its form dictates which plane-wave states are occupied:

$$
\varepsilon_{\mathbf{k}} \equiv \epsilon_{|\mathbf{k}|}-\mu-\Lambda_{\mathbf{k}} \leq 0,
$$

where we have introduced a "mean field" $\Lambda_{\mathbf{k}}$, which is the difference between the "bare" dispersion relation $\epsilon_{k}-\mu$ and the renormalized one.

The mean field $\Lambda_{\mathbf{k}}$ is our variational parameter. This field renormalizes the bare electronic dispersion relation, and therefore the Fermi surface, which is defined as $\varepsilon_{\mathbf{k}}=0$. Minimizing the ground-state energy,

$$
\begin{aligned}
E= & \left\langle\Lambda_{\mathbf{k}}|H| \Lambda_{\mathbf{k}}\right\rangle=\sum_{\mathbf{k}}\left(\epsilon_{\mathbf{k}}-\mu\right) N_{\mathbf{k}} \\
& +\frac{1}{2 \Omega} \sum_{\mathbf{k}, \mathbf{k}^{\prime}} N_{\mathbf{k}} N_{\mathbf{k}^{\prime}}\left[V(0)-V\left(\mathbf{k}-\mathbf{k}^{\prime}\right)\right],
\end{aligned}
$$

with respect to the functional form of $\Lambda_{\mathbf{k}}$ yields a selfconsistency equation that determines $\Lambda_{\mathbf{k}}$. Proceeding as in Ref. 18, we find

$$
\Lambda_{\mathbf{q}}=\frac{1}{\Omega} \sum_{\mathbf{k}}[V(\mathbf{k}-\mathbf{q})-\bar{V}] N_{\mathbf{k}},
$$

where $\bar{V} \equiv \int d^{2} \mathbf{r} V(r)$ and $N_{\mathbf{k}} \equiv\left\langle\psi_{\mathbf{k}}^{\dagger} \psi_{\mathbf{k}}\right\rangle=\Theta\left(-\varepsilon_{\mathbf{k}}\right)$. Note that $\Lambda_{\mathbf{q}}$ coincides with the one-loop Hartree-Fock approximation to the Fermionic self-energy $\Sigma(\mathbf{q}, \omega)$ (the frequency dependence dropping out at this level for static interactions). When the above equation has more than one solution, the one that minimizes

$$
E=\sum_{\mathbf{k}} N_{\mathbf{k}}\left(\varepsilon_{\mathbf{k}}+\frac{1}{2} \Lambda_{\mathbf{k}}\right)
$$

must be chosen. The above expression results from substituting Eq. (8) into Eq. (7). The second term inside the brackets may be interpreted as a double-counting correction to the 
naive mean-field theory which emerges from the variational calculation.

\section{SMALL FERMI SURFACE DEFORMATIONS}

Let us split the mean field $\Lambda_{\mathbf{k}}$ into two parts: a rotationsymmetric part and a symmetry-breaking part. The latter will have a number of components, corresponding to different values of the angular momentum quantum number of the electron-hole pair. Nevertheless near an instability of the isotropic state, or a sufficiently weak first-order transition out of it, we can assume, save accidental degeneracies, that only one of these components is finite. We thus write

$$
\Lambda_{\mathbf{q}}=\Lambda_{0}(q)+\Lambda_{l}(q) \cos \left(l \theta_{\mathbf{q}}\right),
$$

or, equivalently,

$$
\varepsilon_{\mathbf{q}}=\varepsilon_{0}(q)-\Lambda_{l}(q) \cos \left(l \theta_{\mathbf{q}}\right)
$$

where $\varepsilon_{0}(q) \equiv \hbar^{2} q^{2} / 2 m-\mu-\Lambda_{0}(q)$ is the renormalized dispersion relation before the instability sets in and $l$ $=2,3,4, \ldots$ determines the symmetry of the instability (see Fig. 1). We have chosen a particular orientation of the deformation of the Fermi surface without loss of generality.

In the symmetric phase (zero deformation), the Fermi momentum $\hbar k_{F}^{0}$ is defined by

$$
\varepsilon_{0}\left(k_{F}^{0}\right)=0
$$

In the symmetry-broken phase, this quantity depends on the direction of $\mathbf{k}$, given by the angle $\theta$ :

$$
k_{F}^{0} \rightarrow k_{F}(\theta)=k_{F}^{0}+\delta k_{F}(\theta) .
$$

The offset of the Fermi vector is given by

$$
\varepsilon_{0}\left(k_{F}^{0}+\delta k_{F}\right)-\Lambda_{l}\left(k_{F}^{0}+\delta k_{F}\right) \cos (l \theta)=0 .
$$

All results presented in the remaining of this paper have been obtained by solving this equation for small deformations of the Fermi surface, i.e., under the assumption that

$$
\left|\delta k_{F}(\theta)\right| \ll k_{F}^{0}
$$

in all directions $\theta$. In particular we will assume $\delta k_{F}$ to be small enough that the symmetric part of the dispersion relation can be linearized:

$$
\varepsilon_{0}\left(k_{F}^{0}+\delta k_{F}^{0}\right) \approx \hbar v_{F}^{0} \delta k_{F} .
$$

This is quite distinct from the work of other authors, where nonlinear terms in the dispersion relation were invoked to stabilize a quantum critical point. ${ }^{17,36-38}$ That is discussed in detail in Sec. IX. Similarly we will assume that within $\delta k_{F}^{0}$ of the Fermi vector, the $|\mathbf{k}|$-dependent amplitude of the deformation potential can be approximated by a constant

$$
\Lambda_{l}\left(k_{F}^{0}+\delta k_{F}\right) \approx \Lambda_{l}\left(k_{F}^{0}\right) \equiv \Lambda .
$$

Note that we are not writing explicitly the dependence of $\Lambda$ on the angular momentum quantum number, $l$.

The approximations [Eqs. (15) and (16)] are valid when the following conditions hold for $n=1,2,3, \ldots$ :

$$
\frac{1}{(n+1) !} \varepsilon_{0}^{(n+1)} \delta k_{F}^{n}, \quad \frac{1}{n !} \Lambda^{(n)} \delta k_{F}^{n-1} \ll \hbar v_{F}^{0},
$$

where $\Lambda^{(n)} \equiv \partial^{n} \Lambda_{l}(k) /\left.\partial k^{n}\right|_{k=k_{F}^{0}}$ and $\varepsilon_{0}^{(n)} \equiv \partial^{n} \varepsilon_{0}(k) /\left.\partial k^{n}\right|_{k=k_{F}^{0}}$. Under these conditions, Eq. (13) yields the deformation of the Fermi surface as

$$
\delta k_{F}(\theta)=\frac{\Lambda}{\hbar v_{F}^{0}} \cos (l \theta) .
$$

Note that Eq. (18) allows us to rewrite the small-deformation condition [Eq. (14)] in the form

$$
\Lambda \ll \hbar \kappa_{F}^{0} v_{F}^{0} .
$$

Using the decompositions (3) and (10) in the selfconsistency Eq. (8), we can express the self-consistency equation as

$$
\frac{1}{4 \pi^{2}} \int_{0}^{2 \pi} d \theta_{\mathbf{k}} \cos \left(l \theta_{\mathbf{k}}\right) \int_{k_{F}^{0}}^{k_{F}^{0}+\delta k_{F}(\theta)} d k k V_{l}\left(k, k_{F}^{0}\right)=\Lambda .
$$

In general, this is a self-consistency equation determining the values of $\Lambda$ that minimize (and maximize) the energy. The self-consistency comes in through the dependence of $\delta k_{F}^{0}(\theta)$ on $\Lambda$, Eq. (18). Now, the integral Eq. (20) can be reduced to a polynomial equation in $\Lambda$ by invoking condition (13) again to keep a finite number of terms of the expansion of the interaction potential around the Fermi surface:

$$
\begin{aligned}
V_{l}\left(k, k_{F}^{0}\right) \approx & V+V^{\prime}\left(k-k_{F}^{0}\right)+\frac{1}{2} V^{\prime \prime}\left(k-k_{F}^{0}\right)^{2}+\frac{1}{3 !} V^{\prime \prime \prime}\left(k-k_{F}^{0}\right)^{3} \\
& +\ldots
\end{aligned}
$$

Here $V \equiv V_{l}\left(k_{F}^{0}, k_{F}^{0}\right)$ gives the strength of the coupling on the Fermi surface and $V^{\prime} \equiv \partial V_{l}\left(k, k_{F}^{0}\right) /\left.\partial k\right|_{k=k_{F}^{0}}$ $=\partial V_{l}\left(k_{F}^{0}, k\right) /\left.\partial k\right|_{k=k_{F}^{0}}$ is its slope. Higher derivatives give the curvature, etc. Substituting Eq. (21) into Eq. (20) we obtain the following equation:

$$
\begin{aligned}
\Lambda= & \frac{1}{4 \pi^{2}}\left\{V k_{F}^{0} \int_{0}^{2 \pi} d \theta \cos (l \theta) \delta k_{F}(\theta)\right. \\
& +\frac{V+V^{\prime} k_{F}^{0}}{2} \int_{0}^{2 \pi} d \theta \cos (l \theta) \delta k_{F}(\theta)^{2} \\
& \left.+\frac{V^{\prime}}{3} \int_{0}^{2 \pi} d \theta \cos (l \theta) \delta k_{F}(\theta)^{3}+\ldots\right\} .
\end{aligned}
$$

Substituting the value of $\delta k_{F}(\theta)$ given by Eq. (18) and carrying out the integration with respect to $\theta$, we obtain, to a given order in $\delta k_{F} / k_{F}^{0}=\Lambda / \hbar k_{F}^{0} v_{F}^{0}$, a polynomial equation in $\Lambda$.

\section{INSTABILITY EQUATION}

The instability equation is found by solving the selfconsistency Eq. (20) at lowest order in our small parameter expansion, Eq. (21). Using $V_{l}\left(k, k_{F}^{0}\right) \approx V_{l}\left(k_{F}^{0}, k_{F}^{0}\right)$, the critical $V_{l}$ required for the instability is found to be 


$$
V_{l}\left(k_{F}^{0}, k_{F}^{0}\right)=\frac{4 \pi \hbar v_{F}^{0}}{k_{F}^{0}} \equiv V_{\text {crit }} .
$$

This is our instability equation. Note that $V_{l}\left(k_{F}^{0}, k_{F}^{0}\right)$ is the only parameter of the interaction potential entering the instability equation (although the $l=0$ amplitude is also important as it renormalizes the Fermi velocity $\left.v_{F}^{0}\right)$. For $V_{l}\left(k_{F}^{0}, k_{F}^{0}\right)$ $<V_{\text {crit }}$, the symmetric Fermi surface is a (local) energy minimum. For $V_{l}\left(k_{F}^{0}, k_{F}^{0}\right)>V_{\text {crit }}$, it is a maximum.

Writing $V_{l}\left(k_{F}^{0}, k_{F}^{0}\right)$ and $v_{F}^{0}$ explicitly in the above equation, we find it in the form

$$
\frac{\hbar^{2}}{m}=\int_{0}^{\infty} \operatorname{drr} V(r)\left[J_{l}\left(k_{F}^{0} r\right)^{2}-J_{1}\left(k_{F}^{0} r\right)^{2}\right]
$$

for $l=1,2,3, \ldots$. Note that the above equation lacks any solutions with $l=1$, as was found in $D=3,{ }^{18}$ due to the Galilean invariance of the system. ${ }^{34}$

As discussed in Ref. 18 for the three-dimensional case, Eq. (23) is a microscopic version of the Pomeranchuk instability condition, ${ }^{16}$ and Eq. (23) reduces to the Pomeranchuk condition if we use our mean-field theory to compute the Landau Fermi liquid parameters in terms of the microscopic interaction $V\left(\left|\mathbf{k}-\mathbf{k}^{\prime}\right|\right)$ (see Sec. VIII).

\section{ORDERED STATE}

We now discuss the evolution of the amplitude of the deformation, $\Lambda$, in the ordered ground state realized when $V_{m}\left(k_{F}, k_{F}\right)>V_{\text {crit }}$. To describe the ordered state, we will need to go beyond the lowest order in $\delta k_{F} / k_{F}^{0}$ in Eq. (21). To derive the instability Eq. (23), we only used the first parameter in the expansion of the $l^{\text {th }}$ component of the interaction potential, Eq. (21): $V_{l}\left(k, k_{F}^{0}\right) \approx V$. Let us now also keep the first derivative

$$
V_{l}\left(k, k_{F}^{0}\right) \approx V+V^{\prime}\left(k-k_{F}^{0}\right) .
$$

When substituted in the self-consistency equation in the form of Eq. (20), we get, after integrating with respect to $k$, Eq. (22). Substituting Eq. (18) and carrying out the integrals we get

$$
\Lambda=V \frac{k_{F}^{0}}{4 \pi \hbar v_{F}^{0}} \Lambda+\frac{V^{\prime}}{16 \pi \hbar^{3} v_{F}^{0^{3}}} \Lambda^{3} .
$$

This equation admits two solutions: the trivial one, $\Lambda=0$, minimizes the ground-state energy when $V<V_{\text {crit. }}$ On the other hand when $V>V_{\text {crit }}$ the above solution is a maximum. If $V^{\prime}<0$, the minima are at

$$
\frac{\Lambda}{\hbar v_{F}^{0} k_{F}^{0}}= \pm\left(-\frac{4 V_{\text {crit }}}{V^{\prime} k_{F}^{0}}\right)^{1 / 2}\left(\frac{V-V_{\text {crit }}}{V_{\text {crit }}}\right)^{1 / 2} .
$$

The amplitude on the Fermi surface of the deformation potential thus grows in second-order fashion, with critical exponent $=1 / 2$, as expected for this mean-field theory and depicted in Fig. 2(a). If $V^{\prime}>0$, on the other hand, we obtain the unphysical result that the Fermi surface deformation decreases as the instability point is approached from below [see Fig. 2(b)]. This is an indication that linearizing the interac-
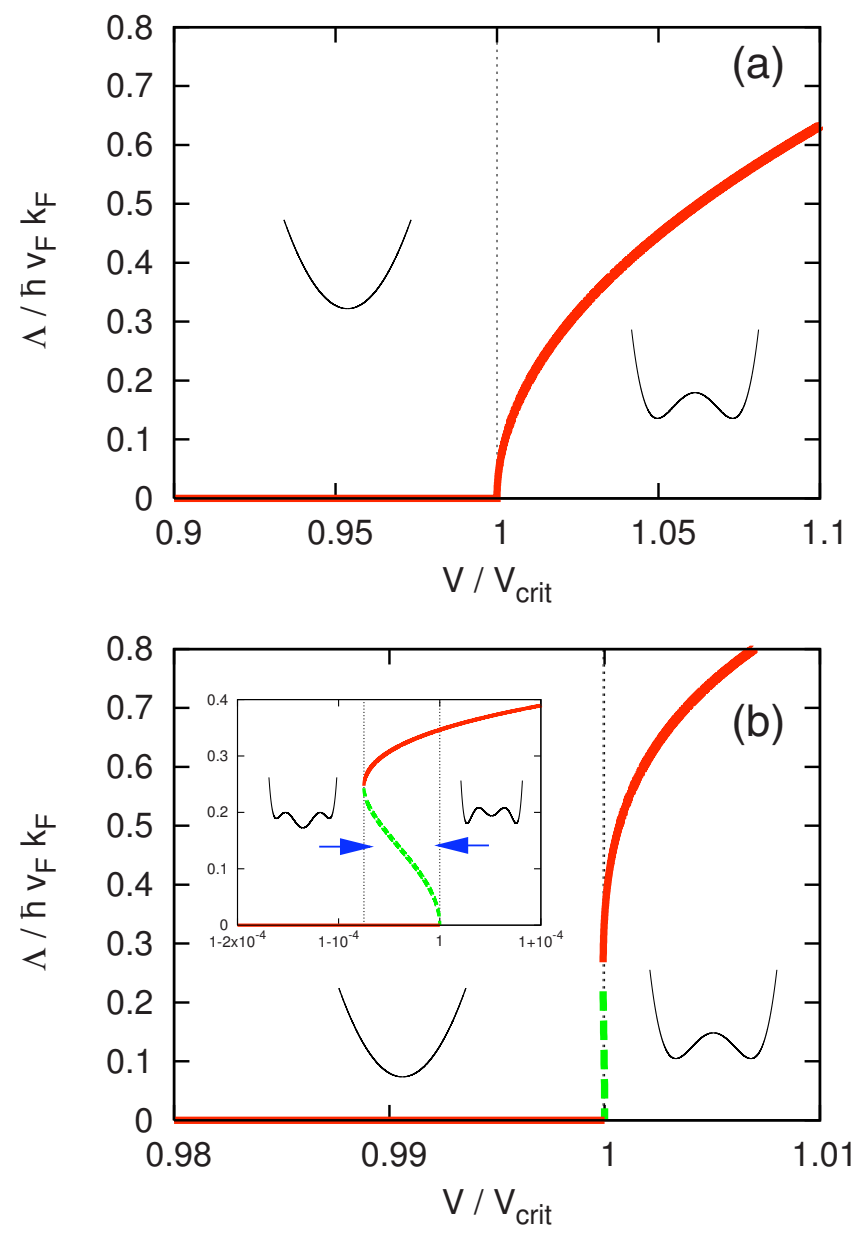

FIG. 2. (Color online) Values of the amplitude on the Fermi surface of the deformation potential, $\Lambda$, that minimize the free energy (solid lines), plotted as functions of the coupling strength in units of its "critical" value, $V / V_{\text {crit }}$. (a) $V^{\prime} k_{F} / V_{\text {crit }}=-1$ and $V^{(n)} k_{F}^{n} / V_{\text {crit }} \approx 0$ for all $n=2,3, \ldots$ [Eq. (27)] (b) $V^{\prime} k_{F} / V_{\text {crit }}=1$, $V^{2} k_{F}^{2} / V_{\text {crit }}=-2+1 / 50, \quad V^{3} k_{F}^{3} / V_{\text {crit }}=-1$, and $V^{(n)} k_{F}^{n} / V_{\text {crit }} \approx 0$ for $n$ $\geq 4$ [Eq. (30)]. The dashed line in panel (b) indicates an additional stationary point, but it is a maximum, not a minimum. The region where the transition takes place has been blown out in the inset. The dotted lines indicate the critical coupling of Eq. (23) [panels (a) and (b)] and the lower bound in Eq. (32) [panel (b)]. The structure of the free energy in the different parameter regions has been sketched for illustration.

tion in Eq. (21) is no longer adequate to describe a transition which potentially is becoming first order. To address this we go beyond the assumption of Eq. (25) in the next section.

\section{FIRST-ORDER QUANTUM PHASE TRANSITION}

Within our linearized theory we have shown that if $V^{\prime}$ $>0$, unphysical solutions occur. These solutions are suggestive of the isotropic state being a local minimum with the true ground state separated from it by a first-order transition, where the Fermi surface jumps to one of lower symmetry. Linearization of $V_{l}\left(k, k_{F}^{0}\right)$ around $k=k_{F}^{0}$, Eq. (25), is therefore not realistic for change that is no longer infinitesimal near the transition. We therefore carry out the expansion (21) to 
the third, rather than first, order. It is useful to introduce the following, dimensionless parameters describing the interaction potential and the resulting deformation of the band structure:

$$
\begin{gathered}
v^{(n)} \equiv \frac{V^{(n)} k_{F}^{0^{n}}}{V_{\text {crit }}}, \\
\lambda \equiv \frac{\Lambda}{\hbar v_{F}^{0} k_{F}^{0}} .
\end{gathered}
$$

In terms of these, the self-consistency equation takes the form

$$
(v-1) \lambda+\frac{1}{4}\left(v^{\prime}+\frac{v^{\prime \prime}}{2}\right) \lambda^{3}+\frac{v^{\prime \prime \prime}}{48} \lambda^{5}=0 .
$$

Taking $v^{\prime \prime} \approx v^{\prime \prime \prime} \approx 0$ and solving for $\lambda$ recovers Eq. (27) as $\lambda= \pm 2\left(-1 / v^{\prime}\right)^{1 / 2}(v-1)^{1 / 2}$. However, Eq. (30) has valid solutions for $v^{\prime}>0$, too. All the solutions are straightforward to obtain analytically. Two examples are plotted in Fig. 2.

We note that there are two types of behavior. For

$$
v^{\prime} o+v^{\prime \prime} / 2<0
$$

(which reduces to $v^{\prime}<0$ in the limit of small $v^{\prime \prime}$ ), there is a second-order transition as we found above. However, when the above condition is not met, there is a range of values of $V$ for which the free energy has a triple-well structure. The instability is then pre-empted by a weakly first-order quantum phase transition-a small "jump" in the shape of the Fermi surface. The value of the coupling strength at which this happens, $V_{\text {jump }}<V_{\text {crit }}$, is bounded by

$$
1+\frac{3\left(v^{\prime}+v^{\prime \prime} / 2\right)^{2}}{4 v^{\prime \prime \prime}} \leq \frac{V_{\text {jump }}}{V_{\text {crit }}}<1 .
$$

The corresponding phase diagram is shown in Fig. 3. The exact location of the jump depends on additional parameters characterizing the fermion-fermion interaction [Specifically, it depends on the off-diagonal second and third derivatives of $V_{l}(k, q)$ with respect to $k$ and $q$.]

The above is only valid for $v^{\prime \prime \prime}<0$. If $v^{\prime \prime \prime}>0$, higher-order terms describing the dependence of $V_{l}(k, q)$ on $k$ and $q$ even further away from the Fermi surface become important. In that case the transition is no longer even weakly first order and the precise behavior of the model depends on more specific details. Naturally as the whole solution relies on the assumption that $\delta k_{F}(\theta)$ is small, the range in which it is reliable is restricted to values of the parameters for which the jump is small.

It is important to stress that the above arguments rely on the approximation of Eq. (18). In the present case, however, the approximation made in that equation requires not only that $\left|\Lambda^{\prime}\right| \ll v_{F}$, but indeed that Eq. (17) holds for $n=1,2,3$. Equivalently we have neglected the additional renormalization of the Fermi velocity and higher-order derivatives of the dispersion relation resulting from the symmetry-breaking part of the Fermi surface deformation, compared to the symmetric contribution.

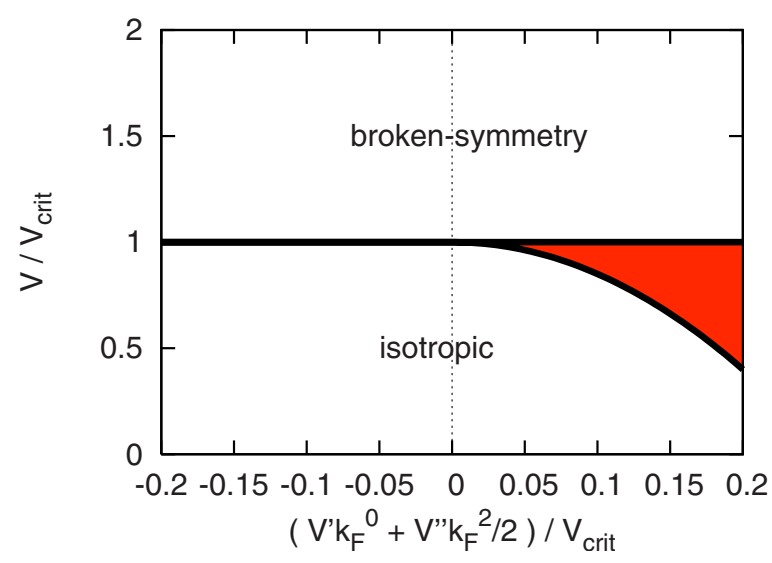

FIG. 3. (Color online) Phase diagram for small symmetrybreaking deformations of the Fermi surface with a given value of the angular momentum quantum number $l$. In the symmetric state, the Fermi surface is circular. In the broken-symmetry state, it has one of the configurations of Fig. 1. In the shaded region one of the two states is stable, and the other metastable, so the transition takes place through a first-order jump. The parameter $V^{\prime \prime \prime} k_{F}^{3} / V_{\text {crit }}$ $=-1 / 20$, which controls the width of this region [Eq. (32)].

\section{LANDAU PARAMETERS}

Variation of Eq. (7) gives the change of the ground-state energy associated with an arbitrary redistribution of the fermions in momentum space, $N_{\mathbf{k}} \rightarrow N_{\mathbf{k}}+\delta N_{\mathbf{k}}$ :

$$
\delta E=\sum_{\mathbf{k}} \varepsilon_{\mathbf{k}} \delta N_{\mathbf{k}}+\frac{1}{2 \Omega} \sum_{\mathbf{k}, \mathbf{k}^{\prime}} f\left(\mathbf{k}, \mathbf{k}^{\prime}\right) \delta N_{\mathbf{k}} \delta N_{\mathbf{k}^{\prime}} .
$$

This coincides with the usual expression from Landau Fermi liquid theory. ${ }^{39}$ The effective interaction between quasiparticles is given by $f\left(\mathbf{k}, \mathbf{k}^{\prime}\right)=\bar{V}-V\left(\mathbf{k}-\mathbf{k}^{\prime}\right)$ in terms of the microscopic parameters. The Landau Fermi liquid parameters can be defined from this function in the usual way:

$$
f\left(\mathbf{k}, \mathbf{k}^{\prime}\right)=\frac{1}{\rho(0)} \sum_{l=0}^{\infty} F_{l} \cos \left[l\left(\theta_{\mathbf{k}}-\theta_{\mathbf{k}^{\prime}}\right)\right] \quad \text { for } k=k^{\prime}=k_{F}^{0},
$$

where $\rho(0)=1 / 2 \pi \hbar v_{F}^{0}$ is the density of states at the Fermi energy. Thus

$$
F_{l}=\rho(0)\left(\delta_{l, 0} \bar{V}-V_{l}\right) .
$$

In terms of these Landau parameters, the Pomeranchuk instability Eq. (23) of Sec. V takes the usual form, $F_{l}<-2$. The microscopic parameters $V^{\prime}, V^{\prime \prime}$, etc. introduced in Sec. VI are related to derivatives of $f\left(\mathbf{k}, \mathbf{k}^{\prime}\right)$ at the Fermi energy and can thus be regarded as generalizations of the Landau parameters.

\section{QUADRUPOLE-QUADRUPOLE INTERACTIONS}

In Ref. 17 Oganesyan, Kivelson, and Fradkin (OKF) introduced an effective Hamiltonian which has been employed widely to study the $l=2$ instability on the continuum. ${ }^{22,23}$ It 
features an anisotropic, "quadrupole-quadrupole" effective interaction. By contrast our Hamiltonian, Eq. (1), features a central interaction potential. In this section we relate the two.

The OKF Hamiltonian is

$$
\begin{aligned}
H_{O K F}= & \int d \mathbf{r} \psi^{\dagger}(\mathbf{r})\left[-\frac{\hbar^{2} \nabla^{2}}{2 m}-\mu\right] \psi(\mathbf{r}) \\
& +\frac{1}{4} \int d \mathbf{r} \int d \mathbf{r}^{\prime} \mathcal{F}_{2}\left(\mathbf{r}-\mathbf{r}^{\prime}\right) \operatorname{tr}\left[\hat{\mathbf{Q}}^{\dagger}(\mathbf{r}) \hat{\mathbf{Q}}\left(\mathbf{r}^{\prime}\right)\right]
\end{aligned}
$$

This corresponds to postulating directly an anisotropic density-density interaction with $l=2$ symmetry, which by analogy with classical liquid crystals, is assumed to be of quadrupole-quadrupole form. ${ }^{40}$ The "quadrupoles" used here are the quadrupole moments of the electronic momentum distribution, ${ }^{17}$

$$
\hat{\mathbf{Q}}(\mathbf{r}) \equiv-\frac{1}{k_{F}^{2}} \psi^{\dagger}(\mathbf{r})\left(\begin{array}{cc}
\hat{\partial}_{x}^{2}-\hat{\partial}_{y}^{2} & 2 \hat{\partial}_{x} \hat{\partial}_{y} \\
2 \hat{\partial}_{x} \hat{\partial}_{y} & \hat{\partial}_{y}^{2}-\hat{\partial}_{x}^{2}
\end{array}\right) \psi(\mathbf{r}) .
$$

The philosophy behind the effective Hamiltonian in Eq. (36) is that in order to describe the important fluctuations on approaching the Pomeranchuk instability, in a given channel $l$, from the isotropic state it is not important to include fluctuations tending to create either distortions of the Fermi surface shape with different symmetry or an inhomogeneous state (backscattering terms). To understand the relationship of theories based on this approximation to our analysis of the generic central Hamiltonian of Eq. (2), we will construct a central Hamiltonian whose form is constrained so that its leading instability has an effective Hamiltonian of the OKF form.

In order to compare to the generic Hamiltonian [Eq. (2)], we first move to reciprocal space

$$
\hat{\mathbf{Q}}(\mathbf{r}) \equiv \sum_{\mathbf{k}_{1} \mathbf{k}_{2}} \psi^{\dagger}\left(\mathbf{k}_{1}\right) Q_{\mathbf{k}_{2}} \psi\left(\mathbf{k}_{2}\right) e^{i\left(\mathbf{k}_{1}-\mathbf{k}_{2}\right) \cdot \mathbf{r}} .
$$

One finds $Q_{\mathbf{k}}^{11}=-Q_{\mathbf{k}}^{22}=-\left(k^{2} / k_{F}^{2}\right) \cos (2 \theta) \quad$ and $\quad Q_{\mathbf{k}}^{12}=Q_{\mathbf{k}}^{21}$ $=\left(k^{2} / k_{F}^{2}\right) \sin (2 \theta)$ so that $\operatorname{tr}\left[Q_{\mathbf{k}_{1}} Q_{\mathbf{k}_{2}}\right]=2\left(k_{1}^{2} k_{2}^{2} / k_{\mathrm{F}}^{4}\right) \cos 2\left(\theta_{1}-\theta_{2}\right)$. Thus

$$
\begin{aligned}
H_{O K F}= & \sum_{\mathbf{k}}\left(\widetilde{\epsilon}_{\mathbf{k}}-\mu\right) \psi_{\mathbf{k}}^{\dagger} \psi_{\mathbf{k}}-\frac{1}{2 \Omega} \frac{(2 \pi)^{4}}{k_{F}^{4}} \\
& \times \sum_{\mathbf{k}, \mathbf{k}^{\prime}, \mathbf{q}} \mathcal{F}_{2}(\mathbf{q})\left(\mathbf{k}-\frac{\mathbf{q}}{2}\right)^{2}\left(\mathbf{k}^{\prime}+\frac{\mathbf{q}}{2}\right)^{2} \\
& \times \cos \left[2\left(\theta_{\mathbf{k}-\mathbf{q} / 2}-\theta_{\mathbf{k}^{\prime}+\mathbf{q} / 2}\right)\right] \\
& \times \psi_{\mathbf{k}+\mathbf{q} / 2}^{\dagger} \psi_{\mathbf{k}^{\prime}-\mathbf{q} / 2}^{\dagger} \psi_{\mathbf{k}-\mathbf{q} / 2} \psi_{\mathbf{k}^{\prime}+\mathbf{q} / 2},
\end{aligned}
$$

where the Fourier transform of the interaction potential is defined by $\mathcal{F}_{2}\left(\mathbf{r}-\mathbf{r}^{\prime}\right) \equiv \Omega^{-1} \Sigma_{\mathbf{q}} e^{i \mathbf{q} \cdot\left(\mathbf{r}-\mathbf{r}^{\prime}\right)} \mathcal{F}_{2}(\mathbf{q})$, and we have introduced the notation

$$
\tilde{\epsilon}_{\mathbf{k}} \equiv \epsilon_{\mathbf{k}}+\frac{(2 \pi)^{4}}{2 \Omega k_{F}^{4}} \sum_{\mathbf{q}} \mathcal{F}_{2}(\mathbf{q})(\mathbf{k}-\mathbf{q})^{2} \mathbf{k}^{2} \cos \left[2\left(\theta_{\mathbf{k}-\mathbf{q}}-\theta_{\mathbf{k}}\right)\right]
$$

Like our Hamiltonian, Eq. (37) features a pairwise interaction that preserves the total momentum of the pair, q. Note, however, the complicated dependence on $\mathbf{q}, \mathbf{k}^{\prime}$, and $\mathbf{k}$. By contrast in Eq. (2) the interaction depends only on the transferred momentum, $\hbar\left(\mathbf{k}-\mathbf{k}^{\prime}\right)$. The dependence on $\mathbf{q}$, in particular, means that the OKF interaction is not uniform. However the actual form of $\mathcal{F}_{2}(\mathbf{q})$ is not very important ${ }^{17}$ and it is customary $^{17,26}$ to take $\mathcal{F}_{2}(\mathbf{q})=\mathcal{F}_{2} \delta_{\mathbf{q}, 0}$. With this assumption Eq. (37) takes the simpler form

$$
\begin{aligned}
H_{O K F}= & \sum_{\mathbf{k}}\left(\widetilde{\epsilon}_{\mathbf{k}}-\mu\right) \psi_{\mathbf{k}}^{\dagger} \psi_{\mathbf{k}}-\frac{1}{2 \Omega} \frac{(2 \pi)^{4}}{k_{F}^{4}} \sum_{\mathbf{k}, \mathbf{k}^{\prime}} \mathcal{F}_{2} \mathbf{k}^{2} \mathbf{k}^{\prime 2} \\
& \times \cos \left[2\left(\theta_{\mathbf{k}}-\theta_{\mathbf{k}^{\prime}}\right)\right] \psi_{\mathbf{k}}^{\dagger} \psi_{\mathbf{k}^{\prime}}^{\dagger} \psi_{\mathbf{k}} \psi_{\mathbf{k}^{\prime}} .
\end{aligned}
$$

Let us now compare this Hamiltonian to the form considered here, given by Eq. (2). In our trial ground state, Eq. (5), the only terms in the triple sum that contribute to the energy have $\mathbf{k}=\mathbf{k}^{\prime}$ or $\mathbf{k}=-\mathbf{k}^{\prime}$. Omitting all other terms from Eq. (2) it takes the simpler form

$$
\begin{aligned}
H= & \sum_{\mathbf{k}}\left(\epsilon_{\mathbf{k}}-\mu\right) \psi_{\mathbf{k}}^{\dagger} \psi_{\mathbf{k}} \\
& +\frac{1}{2 \Omega} \sum_{\mathbf{k}, \mathbf{k}^{\prime}}\left[V\left(\mathbf{k}^{\prime}-\mathbf{k}\right)-V(0)\right] \psi_{\mathbf{k}}^{\dagger} \psi_{\mathbf{k}^{\prime}}^{\dagger} \psi_{\mathbf{k}} \psi_{\mathbf{k}^{\prime}}
\end{aligned}
$$

Substituting in this expression the partial wave expansion of the interaction potential, Eq. (3), we obtain a series of different interaction terms, labeled by $l$, which lead to Fermi surface deformations with different symmetries. Near a secondor weakly first-order Pomeranchuk distortion with $l=2$, only the corresponding term need be considered (see above). Neglecting all the others the Hamiltonian takes the form

$$
\begin{aligned}
H= & \sum_{\mathbf{k}}\left(\epsilon_{\mathbf{k}}-\mu\right) \psi_{\mathbf{k}}^{\dagger} \psi_{\mathbf{k}}+\frac{1}{2 \Omega} \sum_{\mathbf{k}, \mathbf{k}^{\prime}} V_{2}\left(k, k^{\prime}\right) \\
& \times \cos \left[2\left(\theta_{\mathbf{k}}-\theta_{\mathbf{k}^{\prime}}\right)\right] \psi_{\mathbf{k}}^{\dagger} \psi_{\mathbf{k}^{\prime}}^{\dagger} \psi_{\mathbf{k}} \psi_{\mathbf{k}^{\prime}},
\end{aligned}
$$

whose interaction part coincides with that in Eq. (39) if we take

$$
V_{l}\left(k, k^{\prime}\right)=-\frac{(2 \pi)^{4} \mathcal{F}_{2}}{2 k_{F}^{4}} k^{2} k^{\prime 2} .
$$

Thus although the OKF Hamiltonian does not correspond to a central interaction potential, within our theory it would give the same results as a hypothetical central interaction, whose $l=2$ component happens to be given by Eq. (42). Note that inserting Eq. (42) into Eq. (35) we obtain $\mathcal{F}_{2} \propto F_{2}$, as expected. ${ }^{17}$

From this result we note that since $\mathcal{F}_{2}$ is negative at the instability, it follows from Eq. (42) that 


$$
V^{\prime}=-\frac{(2 \pi)^{4} \mathcal{F}_{2}}{k_{F}}>0,
$$

(and that $V^{\prime \prime}>0$ too). This implies that within our mean-field theory, based on a linearized dispersion relation, Eq. (15), the central potential model, which captures the OKF Hamiltonian, is in the parameter regime where the Pomeranchuk instability is actually first order. Indeed to stabilize a quantum critical point for the OKF Hamiltonian, it is essential to include nonlinear terms of the symmetric dispersion relation, $\varepsilon_{0}(k)$, as noted in Refs. 17 and 36-38. Conversely, our complementary approach shows the generic conditions under which a quantum critical point can be stabilized without invoking such nonlinearities of the dispersion relation. This is achieved instead by properly taking into account ultraviolet cutoffs implicit in any given central interaction potential, $V(r)$.

Our results are consistent with other work considering specific microscopic realizations of Fermi surface instabilities, which also found wide regions where the transition is first order. ${ }^{15,26}$ We stress that the main lesson one should extract from this is that the order of the quantum phase transition is a very delicate issue, depending on fine details of the effective interaction and the band structure. Indeed the higher-order terms of the dispersion relation alluded to above modify the coefficients of $\lambda^{3}$ and $\lambda^{5}$ in Eq. (30) (for a detailed analysis, see Ref. 36). For example, a large enough $\delta k_{F}^{3}$ term in the expansion of $\varepsilon_{0}\left(k_{F}^{0}+\delta k_{F}\right)$ can change the sign of the coefficient of $\lambda^{3}$, and hence the order of the transition. Thus a full analysis going beyond present calculations would have to treat nonlinearities in the interaction and the dispersion relation on an equal footing. ${ }^{41}$ Moreover higher-order effects beyond the scope of mean-field theories may well upset this balance one way or the other. Such non-mean-field effects will certainly become important whenever the condition [Eq. (19)] does not hold - e.g., if the phase transition is of first order, but not weakly so.

\section{CONCLUSION}

We have provided a mean-field theory for continuum Pomeranchuk transitions in two dimensions. The theory is expressed in terms of a few pertinent parameters $\left(V, V^{\prime}\right.$, $\left.V^{\prime \prime}, \ldots\right)$ for each angular momentum channel. This makes the theory quite general, and applicable for a wide class of central interactions in which the symmetry breaking is not put in explicitly by hand.

Our main results are Eqs. (31) and (32), which determine the phase diagram in Fig. 3. They apply to any central interaction potential in a two-dimensional continuum, described in terms of a few dimensionless parameters, defined in Eq. (28). Depending on the form of the interaction, our theory may lead either to a first- or second-order quantum phase transition. Thus our approach is complementary to other work, ${ }^{17,36-38}$ where a quantum critical point was stabilized by nonlinear terms in the dispersion relation.

A continuum theory is useful for several reasons. One is the direct relevance to several experimental systems, where a Pomeranchuk transition might be realized, for example: 2D electron layers at semiconductor heterojunctions or on liquid helium; layered helium systems ${ }^{42}$ (where a new phase intervening between the Fermi liquid and Mott insulator states has been observed ${ }^{43}$ ); or in a cold-atom setting with trapped fermionic atoms with dipolar repulsion. These are situations where underlying lattice structures are not expected to play a role. Theoretically, continuum Pomeranchuk transitions are fascinating because the resulting broken symmetries are remarkable. While some effects of the nematic symmetry (broken $O(2) / Z_{2}$ ) have already been explored, ${ }^{17}$ we believe there are further implications, for example, effects of nematic halfvortex excitations in such a medium. ${ }^{44}$ Our framework also provides a description of transitions in higher- $l$ channels [leading to $O(2) / Z_{l}$ symmetry broken states], which presumably leads to a broader class of interesting excitations and properties. Some of these issues are currently under investigation.

\section{ACKNOWLEDGMENTS}

J.Q. acknowledges support by CCLRC (now STFC) in association with St. Catherine's College, Oxford. M.H. thanks the European Science Foundation (INSTANS program) for funding a visit to Birmingham and RAL for this work. The authors are grateful to D. G. Barci, E. Fradkin, J. T. Chalker, C. Hooley, I. Paul, B. J. Powell, and M. B. Silva Neto for useful discussions. J.Q. thanks the University of Birmingham for hospitality during the preparation of part of this manuscript. *j.quintanilla@rl.ac.uk

${ }^{1}$ E. Fradkin, S. A. Kivelson, E. Manousakis, and K. Nho, Phys. Rev. Lett. 84, 1982 (2000).

${ }^{2}$ O. Ciftja and C. Wexler, Phys. Rev. B 65, 205307 (2002).

${ }^{3}$ Q. M. Doan and E. Manousakis, Phys. Rev. B 75, 195433 (2007).

${ }^{4}$ C. M. Lapilli and C. Wexler, Phys. Rev. B 73, 085314 (2006).

${ }^{5}$ L. Radzihovsky and A. T. Dorsey, Phys. Rev. Lett. 88, 216802 (2002).

${ }^{6}$ C. Wexler and A. T. Dorsey, Phys. Rev. B 64, 115312 (2001).

${ }^{7}$ E. Fradkin and S. A. Kivelson, Phys. Rev. B 59, 8065 (1999).
${ }^{8}$ A. H. MacDonald and M. P. A. Fisher, Phys. Rev. B 61, 5724 (2000).

${ }^{9}$ K. Yang, arXiv:cond-mat/0508484 (unpublished).

${ }^{10}$ S. A. Kivelson, E. Fradkin, and V. J. Emery, Nature (London) 393, 550 (1998).

${ }^{11}$ S. A. Kivelson, E. Fradkin, and T. H. Geballe, Phys. Rev. B 69, 144505 (2004).

${ }^{12}$ C. J. Halboth and W. Metzner, Phys. Rev. Lett. 85, 5162 (2000).

${ }^{13}$ W. Metzner, D. Rohe, and S. Andergassen, Phys. Rev. Lett. 91, 066402 (2003).

${ }^{14}$ L. Dell'Anna and W. Metzner, Phys. Rev. B 73, 045127 (2006). 
${ }^{15}$ H. Yamase, V. Oganesyan, and W. Metzner, Phys. Rev. B 72, 035114 (2005).

${ }^{16}$ I. Ia. Pomeranchuk, Sov. Phys. JETP 35, 524 (1958).

${ }^{17}$ V. Oganesyan, S. A. Kivelson, and E. Fradkin, Phys. Rev. B 64, 195109 (2001).

${ }^{18}$ J. Quintanilla and A. J. Schofield, Phys. Rev. B 74, 115126 (2006).

${ }^{19}$ K. Yang, arXiv:cond-mat/0502270 (unpublished).

${ }^{20}$ J. Nilsson and A. H. Castro Neto, Phys. Rev. B 72, 195104 (2005).

${ }^{21}$ M. J. Lawler, D. G. Barci, V. Fernandez, E. Fradkin, and L. Oxman, Phys. Rev. B 73, 085101 (2006).

${ }^{22}$ H.-Y. Kee, Phys. Rev. B 67, 073105 (2003).

${ }^{23}$ Y. B. Kim and H.-Y. Kee, J. Phys.: Condens. Matter 16, 3139 (2004).

${ }^{24}$ H.-Y. Kee and Y. B. Kim, Phys. Rev. B 71, 184402 (2005).

${ }^{25}$ H.-Y. Kee, E. H. Kim, and C.-H. Chung, Phys. Rev. B 68, 245109 (2003).

${ }^{26}$ I. Khavkine, C.-H. Chung, V. Oganesyan, and H.-Y. Kee, Phys. Rev. B 70, 155110 (2004).

${ }^{27}$ H. Doh, N. Friedman, and H. Y. Kee, Phys. Rev. B 73, 125117 (2006).

${ }^{28}$ Congjun Wu and Shou-Cheng Zhang, Phys. Rev. Lett. 93, 036403 (2004).

${ }^{29}$ Congjun Wu, Kai Sun, Eduardo Fradkin, and Shou-Cheng
Zhang, Phys. Rev. B 75, 115103 (2007).

${ }^{30}$ S. Stintzing and W. Zwerger, Phys. Rev. B 56, 9004 (1997).

${ }^{31}$ J. Quintanilla, B. L. Gyorffy, J. F. Annett, and J. P. Wallington, Phys. Rev. B 66, 214526 (2002).

${ }^{32}$ P. Nozieres and S. Schmitt-Rink, J. Low Temp. Phys. 59, 195 (1985).

${ }^{33}$ P. Nozieres, J. Phys. I 2, 443 (1992).

${ }^{34}$ P. Wolfle and A. Rosch, J. Low Temp. Phys. 147, 165 (2007).

${ }^{35}$ A. F. Ho and A. J. Schofield, arXiv:0706.1955 (unpublished).

${ }^{36}$ D. G. Barci and L. E. Oxman, Phys. Rev. B 67, 205108 (2003).

${ }^{37}$ E. A. Kim, M. J. Lawler, S. Vishveshwara, and E. Fradkin, Phys. Rev. B 74, 155324 (2006).

${ }^{38}$ D. G. Barci, M. Trobo, V. Fernandez, and L. Oxman, arXiv:0801.4075, Phys. Rev. B (to be published).

${ }^{39}$ See, for example, A. J. Leggett, Rev. Mod. Phys. 47, 331 (1975).

${ }^{40}$ See, for example, Sec. 45 in P. M. Chaikin and T. C. Lubensky, Principles of Condensed Matter Physics (Cambridge University Press, Cambridge, England, 1995).

${ }^{41}$ D. G. Barci (private communication).

${ }^{42}$ H. Godfrin and H.-J. Lauter, in Progress in Low Temperature Physics, edited by W. P. Halperin (North Holland, Amsterdam, 1995), Vol. 14, p. 213-314.

${ }^{43}$ M. Neumann, J. Nyeki, B. Cowan, and J. Saunders, Science 317, 1356 (2007).

${ }^{44}$ D. L. Stein, Phys. Rev. B 18, 2397 (1978). 